\title{
Infantile cerebellar-retinal degeneration
}

INSERM

\section{Source}

INSERM. (1999). Orphanet: an online rare disease and orphan drug data base. Infantile cerebellar-retinal degeneration. ORPHA:313850

Infantile cerebellar-retinal degeneration is a rare, neurodegenerative disorder characterized by an early onset of truncal hypotonia, variable forms of seizures, athetosis, severe global developmental delay, intellectual disability and various ophthalmologic abnormalities, including strabismus, nystagmus, optic atrophy and retinal degeneration. 